\title{
TEMA 3-2016: Papilomatosis respiratoria: Nuevas terapias adyuvantes
}

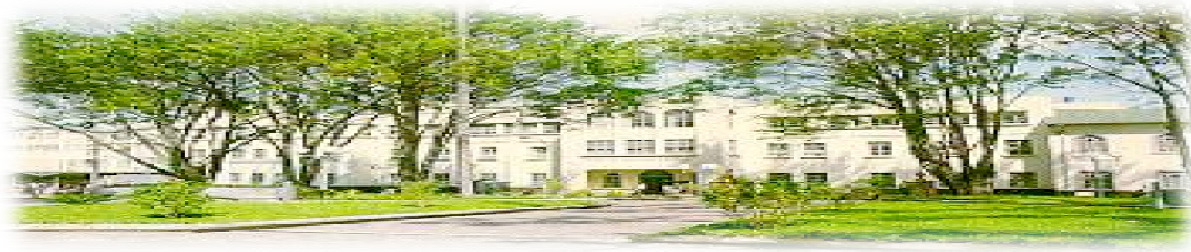

Hospital San Juan de Dios, San José, Costa Rica. Fundado en 1845
Recibido:
$27 / 11 / 2015$
Aceptado:
$13 / 12 / 2015$

Adriana Valverde Zúñiga ${ }^{1}$ Milena Zamora ${ }^{2}$

\begin{abstract}
${ }^{1}$ Médico General. Universidad de Costa Rica, Hospital San Juan de Dios. Correo electrónico: adri0719@hotmail.es

${ }^{2}$ Médico Especialista en Otorrinolaringología. Hospital San Juan de Dios. Docente académica de la unidad de enseñanza de la Escuela de Medicina de la Universidad de Costa Rica.
\end{abstract}

\section{RESUMEN}

La papilomatosis respiratoria(PR) constituye una entidad causada por el Virus del papiloma humano (VPH), la cual, a pesar de ser poco frecuente, continúa siendo la principal neoplasia laríngea en la población pediátrica y una de las causas a descartar ante la presencia de estridor y ronquera. A pesar de que el tratamiento quirúrgico continúa siendo el principal pilar de esta patología, múltiples tratamientos adyuvantes han surgido en los últimos años con el objetivo de mejorar la calidad de vida, disminuir los síntomas, disminuir la cantidad de intervenciones quirúrgicas y alcanzar la remisión de la enfermedad. Esta revisión pretende analizar las principales tendencias actuales de la terapia adyuvante.

\section{PALABRAS CLAVE}

Papilomatosis respiratoria, adjuvante, cidofovir, bevacizumab, indol 3-carbinol, interferón 2-alfa pegilado.

\begin{abstract}
Respiratory papillomatosis is a disease caused by the papilloma human virus. Although infrequent, it is the most common cause of benign larynx neoplasm in the pediatric population, and it is one of the first differential diagnoses of stridor and hoarseness in children. Although surgical therapy continues to be its main treatment, adjuvant therapy has been implemented during the last few decades with the purpose of reducing the number of surgical interventions and achieving remission of the disease. The main objective of this paper is to analyze such adjuvant therapy and its benefits.
\end{abstract}

ISSN 


\section{KEY WORDS}

Respiratory papillomatosis, adjuvant medical therapy,Bevacizumab,Indole 3 carbinol, Cidofovir,Pegylated Interferon Alpha $2 \mathrm{a}$

\section{INTRODUCCIÓN}

La papilomatosis respiratoria consiste en lesiones verrucosas exofíticas situadas en la mucosa de la vía aérea. ${ }^{(1)}$

La papilomatosis respiratoria recurrente (PRR) hace referencia al crecimiento repetido de estas lesiones a cualquier nivel de la vía aérea, siendo el lugar más frecuente la laringe. ${ }^{(2,3)}$

Literatura reciente sitúa esta entidad como la principal causa de neoplasia pediátrica de la vía aérea (VA) con una incidencia estimada de 4.3/100000. ${ }^{(4,5)}$

La PR presenta una distribución bimodal, considerándose de tipo juvenil (PRRJ) si su inicio es antes de los 18 años con un pico a los 5 años yde tipo adulto (PRRA) si su inicio es posterior a los 18 años, con un pico de incidencia entre los 20-30 años. ${ }^{(6)}$ Esta revisión pretende analizar las principales terapias adyuvantes en la actualidad.

\section{ETIOLOGIA}

A pesar de que los principales subtipos involucrados de virus del papiloma humano (VPH) son el 6 y el 11, se han descrito en la literatura otros subtipos como $16,18,31,33$ y $51 .{ }^{(7)}$ La principal ruta de contagio en el caso de PRRJ es la trasmisión vertical, representada por el canal vaginal infectado en pacientes primíparas adolescentes ${ }^{(3,8)}$. La transmisión durante el sexo oral se considera que es la principal ruta para el establecimiento de la misma durante la edad adulta. ${ }^{(6)}$

\section{CUADRO CLÍNICO}

A pesar de que la PR es una patología de naturaleza benigna, esta presenta un carácter progresivo, causando ronquera, estridor e incluso obstrucción de la vía aérea que puede cursar con distrés respiratorio. ${ }^{(3,9)}$ Otras formas de presentación incluyen la tos crónica, neumonía recurrente, falla para progresar, disnea y disfagia. ${ }^{(10)}$ La mayoría de las veces, se asocia a una velocidad de crecimiento lenta, por lo que sus manifestaciones clínicas son insidiosas
(11). Dentro de las principales patologías con las que se realiza diagnóstico diferencial se menciona asma, bronquitis, infección de vías respiratorias superiores y laringitis aguda. El diagnóstico se realiza con una endoscopia de vía aérea, siendo este el método más confiable. ${ }^{(12)}$

Generalmente la PRRJ presenta una severidad muy variable, definiéndose la misma con base en la frecuencia de los procesos quirúrgicos requeridos, alcanzándose en algunos casos la remisión, mientras que en otros casos se ha documentado incluso la necesidad de varios procedimientos quirúrgicos al mes. El Registro Nacional para Papilomatosis Juvenil Recurrente de los Estados Unidos, realizó un estudio con 603 pacientes con una edad media de diagnóstico de 3.1 años, y documentó que aquellos niños diagnosticados antes de los 4 años eran sometidos a mayor cantidad de intervenciones quirúrgicas. Asimismo, este estudio concluyó que el diagnóstico a una menor edad se asociaba con mayor cantidad de sitios involucrados con enfermedad, presentando mejor pronóstico los niños mayores en los que se documentaron lesiones en vía aérea superior. En cuanto a la traqueotomía, se documentó que esta principalmente se realiza en pacientes con enfermedad progresiva y se recomienda solamente en caso de que sea mandatoria, ya que se ha documentado que ayuda a la expansión de la enfermedad a nuevos sitios. ${ }^{(2,9)}$

\section{RIESGO DE MALIGNIZACIÓN}

La PRR se ha asociado con transformación maligna en cerca del $4 \%$ de los pacientes, ocurriendo una integración del virus a células susceptibles por mutaciones en oncogenes como el p53. Los principales factores de riesgo para la transformación maligna son fumado, mutaciones del p53, irradiación, y uso de dro-

\begin{tabular}{|l|}
\hline Cuadro I. Criterios para uso de terapia adyuvante \\
\hline Más de 4 procedimientos quirúrgicos por año \\
\hline $\begin{array}{l}\text { Rápido crecimiento de las lesiones con compromi- } \\
\text { so de la vía aérea }\end{array}$ \\
\hline Propagación de la enfermedad distalmente \\
\hline
\end{tabular}

Fuente: adaptado de Arch Otolaryngol Head Neck Surg. 2010; 136(6):561-565

gas citotóxicas. ${ }^{(12)}$ 
En un estudio realizado en Noruega, en los departamentos de otorrinolaringología (ORL) del Hospital Universitario de Oslo y del Hospital Diaconal de Lovisenberg durante los años 19872009, que incluyó un total de 224 participantes, los cuales fueron divididos con base en la edad de los mismos al momento de realizar el diagnóstico en PRRJ y PRRA. Dicho estudio documentó una mayor prevalencia de VPH 6 (55$90 \%)$ comparado con el VPH 11 (5-42\%). Sin embargo, se documenta una mayor incidencia de este último en la PRRJ al compararla con la PRRA. Además, se determinó una mayor incidencia de malignidad en aquellos pacientes con biopsia negativa por VPH, asociandose la misma con un riesgo 50 veces mayor de desarrollar carcinoma escamoso de vía aérea. Para los pacientes VPH positivos, dicho estudio no documentó un incremento significativo de displasia de alto grado o carcinoma. ${ }^{(6)}$ Además, la literatura hace referencia al hecho de que el subtipo 11 se presenta de manera más agresiva en comparación con el subtipo 6 .

\section{TRATAMIENTO}

El tratamiento de la PRR continúa siendo la citorreducción mediante cirugía, situando como principal objetivo el mantenimiento de una vía aérea permeable y patente, la mejoraría de la función de las cuerdas vocales y lograr la remisión de la enfermedad. ${ }^{(4,10)}$. Es bien conocido que esta patología puede requerir múltiples intervenciones por año, especialmente en la población pediátrica, por lo que se han iniciado estudios sobre terapias adyuvantes que ayuden a minimizar las intervenciones quirúrgicas.

El uso de estas terapias adyuvantes está basado en una serie de criterios, dentro de los que destacan: la realización de más de 4 procedimientos quirúrgicos por año, el rápido crecimiento de las lesiones con compromiso de la vía aérea, o la propagación de la enfermedad distalmente ${ }^{(13)}$. Derkay estableció un sistema de estadiaje basado en el área de superficieinvolucrada, severidad, extensión de distrés respiratorio y calidad de la voz, que permite evaluar la eficacia de los tratamientos emergentes ${ }^{(10)}$.

Bevacizumab:
Constituye un anticuerpo monoclonal humanizado que se une y neutraliza la actividad del factor de crecimiento vascular endotelial. Estudios han demostrado Se ha documentado la presencia de dicho receptor en especímenes laríngeos tomados de niños con PRR. ${ }^{(13)}$

Se han documentado efectos adversos relacionados con el uso de bevacizumab intravenoso, como sangrado, formación de coágulos, elevación de la presión arterial, problemas en la cicatrización,yperforación gastrointestinal, entre otros. Los estudios demuestran que la dosis segura en adultos es de 5-7mg. En un estudio realizado enel Massachusetts Eye and Ear Infirmary Hospital que incluyó 3 pacientes entre los tres y los seis años, se propuso el uso de $1.25 \mathrm{mg}$, extrapolando la experiencia oftalmologica de utilizar dicha dosis de manera intraocular sin asociar efectos adversos. Sin embargo, el mismo señala que dentro de los posibles efectos adversos a nivel local, se sitúa la cicatrización de las cuerdas vocales, respuesta inflamatoria aguda local y obstrucción aguda de la vía aérea. A pesar de que este estudio incluyó solamente tres pacientes, sí se documentó una prolongación deltiempo entre tratamientos quirúrgicos y una disminución del número de tratamientos por año. Este estudio culmina recomendando el uso de Bevacizumab cuando el paciente presente alguno de los siguientes criterios: más de 4 intervenciones por año, no haya respondido a tres ciclos de cidofovir, cuando la familia no desee utilizar este último como primera línea. Sin embargo, menciona que son necesarios más estudios para determinar el número de aplicaciones de esta terapia. ${ }^{(13)}$

Por otro lado, el hospital de Cincinnati en el 2014 realizó un estudio prospectivo que incluyó un total de 9 pacientes con una edad media de 8 años en los que se utilizó citorreducción con KTP laser, más inyecciones subepiteliales de bevacizumab $\quad(25 \mathrm{mg} / \mathrm{ml})$, aplicando 5 inyecciones en un periodo de 4-6 semanas. Contrario al estudio realizado por el Massachusetts Eye and Ear Infirmary Hospita, se utilizaron altas dosis de bevacizumab con el propósito de maximizar la eficacia pero tratando de limitar al máximo los efectos adversos. Este estudio determinó que existe un posible beneficio documentado con respecto a la calidad de voz sin asociar incremento de efectos adversos 
en los pacientes, que debe ser evaluado más a fondo en estudios a futuro. ${ }^{(4)}$

En la actualidad, la dosis autorizada para PRR como terapia adyuvante es de $12.5 \mathrm{mg} .{ }^{(8)}$

\section{Terapia antiretroviral:}

Hace referencia tanto a los agentes utilizados de manera sistémica como lo son el aciclovir y la ribavirina, como a los usados de manera local, como el cidofovir. Este último constituye el principal tratamiento adyuvante en PRR. Fueutilizado

en papilomatosis respiratoria recurrente por primera vez en 1995 por Van Cutsem y se ha observado que induce apoptosis en las células VPH positivas. Es un nucleósido análogo de la deoxycitadina monofosfato que al incorporarse al ADN inhibe a la ADN polimerasa viral, previniendo por tanto la replicación viral. ${ }^{(14)}$ Este fármaco se encuentra aprobado por la FDA para el tratamiento de retinitis por citomegalovirus ya que presenta actividad contra los virus de la familia herpes. $(3,5,10,15)$

Dentro de los efectos adversos citados en la literatura de este grupo de fármacos destacan náuseas, dolor abdominal, vómitos, hepatitis, neutropenia y falla renal aguda. El principal efecto adverso del cidofovir es la nefrotoxicidad, además, se ha asociado con carcinogénesis en estudios in vitro en ratones, lo cual no se ha podido documentar en seres humanos. ${ }^{(10,16)}$

Una revisión sobre el uso de terapia antiviral en pacientes con PRR, realizada por Cochrane, que situó como puntos primarios del tratamiento con cidofovir, la mejoría en la calidad de vida y de los síntomas, y como puntos secundarios se establecieron la reducción en la mortalidad, la disminución en el número de intervenciones, la reducción en el número de días de hospitalización, la disminución endoscópica de la enfermedad y los efectos adversos de los antivirales. En esta se demostró una mejoría de la escala de severidad de Derkay tanto con el uso del cidofovir como con el placebo; se cree que esto se relacionada con la historia natural de la enfermedad, en la cual hay mejoría de la severidad a través del tiempo. A pesar de dicha mejoría, no se documentó beneficio sobre la calidad de vida, por lo que se estima que la escala de Derkay tiene escasa sensibilidad en este parámetro. ${ }^{(10)}$

Se ha hablado recientemente incluso del uso cidofovir inhalado, no obstante son necesa- rios más estudios formales al respecto debido a que la literatura menciona únicamente dos casos. El primero, en el 2006, en un paciente refractario tanto a cirugía como al uso del cidofovir intralesional y de interferón, en el cual se documentó resolución de la patología y se notó como principal complicación hemoptisis. El otro caso reportado constituye un paciente que igualmente presentó mejoría y remisión posterior al uso del cidofovir inhalado, pese a esto, no se puede establecer una relación causal y son necesarios más estudios al respecto. ${ }^{(14,16)}$

Hay ciertos puntos que continúan siendo controversiales con respecto al uso del cidofovir. No existen guías sobre la dosis ni frecuencia necesarias. Se recomiendan, con base en los estudios realizados, dosis en un rango de $2-7 \mathrm{mg} / \mathrm{ml}$ con una dosis máxima de $3 \mathrm{mg} / \mathrm{kg} .{ }^{(8,17)}$. Así mismo el intervalo de aplicación no está definido. La vida media del cidofovir es de 17-65 horas, por lo que se considera que una inyección cada 3-4 días sería lo ideal. La literatura menciona un mayor beneficio en aquellos casos donde se utiliza en periodos entre las 2-4 semanas, comparado con aquellos distanciados un mes entre sí, siendo el número de dosis aún tema de discusión. (17)

Indol 3 carbinol:

Este compuesto es derivado de vegetales de la familia Crucifarae. Estudios han reportado que este compuesto disminuye el riesgo de cáncer hormonodependiente debido que induce un cambio en el metabolismo de la estrona a nivel hepático que produce 2-hidroxiestrona en lugar de 16-hidroxiestrona. Esta última ha probado ser genotóxica e induce cambios en el $\mathrm{ADN}$ que se asocian con una hiperproliferación celular, por lo que se cree que esto inhibe el crecimiento del papiloma.

En un estudio prospectivo realizado en la Universidad de Pittsburg, que incluyó un total de 33 pacientes con una edad promedio de 48 años, documentó que un $70 \%$ de los participantes presentaba respuesta positiva con el uso del IC-3. Sin embargo, la respuesta observada fue mejor en adultos que en niños. Este estudio además documentó que la mejoría se produce rápidamente y que la recurrencia de la enfermedad sucede a largo plazo (más de 5 años). ${ }^{(18)}$

Son necesarios más estudios con respecto a esta terapia que presenta entre sus ventajas ser bien tolerada, no invasiva, y eficaz. ${ }^{(18)}$ 
Interferón gamma:

La activación de macrófagos y monocitos así como la secreción de citoquinas aumenta la respuesta Th1 y la inmunidad adquirida, lo cual ayuda a eliminar las infecciones virales. Se ha considerado que el uso sinérgico de interferón gamma pegilado y el factor estimulante de colonias de granulocitos puede aumentar la respuesta inmune. (19)

Desde 1991 se demostró beneficio con el uso de interferón. En un estudio publicado en New England Journal of Medicine, que incluyó 66 pacientes que fueron distribuidos de manera aleatorizada en dos grupos, el primero recibió interferon mientras que el segundo solamente fue observado. Dicho estudio documentó una mejoría en el $79 \%$ de los pacientes recibiendo tratamiento con interferon, logrando una remisión completa en un $37 \%$ de ellos. ${ }^{(20)}$

En 1995, un estudio retrospectivo realizado en 34 pacientes a los que se les brindó tratamiento con interferon demostró resultados prometedores, con una mejoría definida como una disminución del volumen y de las áreas involucradas mediante evaluación endoscopica, documentada en el $86 \%$ de los pacientes y remisión completa en un $57 \%$ de los mismos. Dentro de los principales efectos adversos asociados al uso de dicha terapia destacan fiebre, letargia, cefalea, elevación de transaminasas y leucopenia. ${ }^{(21)}$

Según un estudio realizado en el 2005 y 2006 por Suter-Montano et al, en 11 pacientes con papilomatosis adulta respiratoria recurrente, en el que se utilizó Interferón pegilado alfa $2 \mathrm{a}$ en una dosis de $180 \mathrm{mcg} / \mathrm{semana}$ por 6 meses y factor estimulante de colonias de granulocitos a $400 \mathrm{mcg} / \mathrm{semana}$ a partir del $3 \mathrm{er}$ mes, por $2 \mathrm{me}-$ ses; se determinó una mejoría significativa de la calidad de vida de los pacientes, así como una disminución de las intervenciones quirúrgicas. Dicho estudio demuestra que esta terapia adyuvante disminuye la recurrencia así como la necesidad de cirugía asociando una mejoría en la calidad de vida. ${ }^{(20)}$

Sin embargo, son necesarios más estudios con mayor población para obtener resultados concluyentes. ${ }^{(19)}$

\section{Otras terapias adyuvantes.}

La literatura menciona el uso de otras terapias tales como la isotretionina o derivados del ácido retinoico, el metrotexate y el control de la enfer- medad por reflujo gastroesofágico. Es necesario continuar realizando estudios que respalden el uso de dichas terapias. ${ }^{(8,21)}$

\section{CONCLUSIONES}

La papilomatosis respiratoria recurrente es una patología que causa un impacto importante en la calidad de vida de los pacientes que la sufren. La exposición a procedimientos quirúrgicos repetitivos se asocia a secuelas tanto a nivel psicológico y social como funcional. Durante los últimos años, se ha trabajado en la implementación de terapias adyuvantes que disminuyan la tasa de recurrencia y aumenten la remisión de dicha enfermedad, evitando las múltiples cirugías que conllevan lesiones y afectan la calidad de vida de estos pacientes. El cidofovir, bevacizumab e interferón han demostrado mediante diversos estudios, la mejoría en la evolución de dicha enfermedad, sin embargo los mismos presentan una casuistica muy variada, con pequeñas poblaciones que dificultan la obtención de datos conclueyentes de los mismos. El interferon es la opción con estudios de mayor tamaño, que ha demostrado una disminución en la necesidad de procedimientos quirurgicos asi como mayor tasa de remisión de la enfermedad. Sin embargo, a pesar de que faltan estudios al respecto que permitan definir el algoritmo de manejo más efectivo de dicha patología, los datos actuales muestran resultados prometedores, estando la evidencia a favor del uso de la adyuvancia en esta patología.

\section{BIBLIOGRAFÍA}

1. Donne A, Clarke R. Recurrent respiratory papillomatosis: an uncommon but potentially devastating effect of human papillomavirus in children. International Journal of STD \& AIDS 2010; 21: 381-385

$2 . \quad$ Reeves W et al. National Registry for JuvenileOnset Recurren t Respiratory Papillomatosis. Arch Otolaryngol Head Neck Surg. 2003;129: 976-982

3. Tasca R, McCormick M, Clarke R. Briish Association of Paediatric Otorhinolaryng ogy members experience with recurrent respirato 
ry papillomatosis. International Journal of Pediat ric Otorhinolaryngology 2006; 70:1183-1187

4. Sidell D et

al. HighDose Sublesional Bevacizumab (Avastin) for Pediatric Recurrent Respiratory Papillomato sis. Annals of Otology, Rhinology \& Laryngolog y. 2014; 123(3): 214-221

5. Chung B, Akst L, Koltai P. 3.5-Year follow-up of intralesional cidofovir protocol for pediatric recurrent respiratory papillomatosis. International Journal of Pediatric Otorhinolaryngology. 2006; 70: 1911-1917

6. Omland T et

al. Recurrent Respiratory Papillomatosis:

HPV Genotypes and Risk of High-

Grade Laryngeal Neoplasia. PLOS ONE. 2014; 9(6): 1-7.

7. Hall J et al. Natural Progression of Dysplasia in Adult Recurrent Respiratory Papillomatosis. Otolaryngology- $\square$ Head and Neck Surgery $\square$ 2011; 144(2) : 252-256

8. Carifi $\mathrm{M}$ et al. Recurrent respiratory papillomatosis: current and future perspectives. Therapeutics and Clinical Risk Management 2015; $11: 731-738$

9. Correia S, Dionisio J, Duro da Costa J. Recurrent respiratory papillomatosis of the airway: The experience of an endoscopic unit. Rev Port Pneumol. 2015; 21(2):82-89

10. Chadha N, James A. Adjuvant antiviral therapy for recurrent respiratory papillomatosisCochrane Database of Systematic Reviews 2010; 1: 1-14.

11. Rojo P et

al. Papilomatosis respiratoria recurrente: una $c$ ausa de dificultad respiratoria progresi-

va. Anales Españoles De Pediatría. 2001; 55(6): 558-560.

12. Fusconi $\mathrm{M}$ et al. Recurrent respiratory papillomatosis by HPV: review of the literature and update on the use of cidofovir. Acta Otorhinolaryngol Ital 2014; 34: 375-381

13. Maturo S, Hartnick J. Use of 532nm Pulsed Potassium Titanyl Phosphate Laser and Adjuvant Intralesional Bevacizumab for Agg res- sive Respiratory Papillomatosis in Children.Arc h Otolaryngol Head Neck Surg. 2010; 136(6):561-565

14. Cespedes M et al. Adult-Onset Recurrent Respiratory Papillomatosis: A Review of Disease Pathogenesis and Implications for Patient Counseling. JAMA Otolaryngol Head Neck Surg. 2015;141(1):78-83

15. Palomar V et

al. Actividad del cidofovir en

la papilomatosis respiratoria infantil recidivante Acta Otorrinolaringol Esp 2005; 56: 22-24

16. Ksiazek J et al. Inhaled Cidofovir as an Adjuvant Therapy for Recurrent Respiratory Papillomatosis. Otolaryngology- $\square$ Head and Neck Surgery $\square$. 2011. 144(4) : 639-641 $\square$ 17. Durvasula V, Richter G. Intralesional cidofovir as adjuvant for the successful management of aggressive respiratory papillomatosis in an infant. International Journal of Pediatric Otorhinolaryngology. 2013; 77: 1912-1915

18. Rosen C, Bryson P. Indole-3-Carbinol for Recurrent Respiratory Papillomatosis: LongTerm Results. Journal of Voice. 2004; 18(2):

248-253

19. Suter-Montano T et

al. Adult Recurrent Respirator Papillomatosis: A New Therapeutic Approach with Pegylated Interf eron Alpha 2 a (Peg-IFNa-2a) and GM-

$C S F$. Otolaryngology- $\square$ Head and Neck Surgery $\square$. 2013; 148(2) 253-260

20. Leventhal GB et al. Long-term response of recurrent respiratory papillomatosis to treatment with lymphoblastoid interferon alfanl.N Engl J Med 1991; 325:613-7.

21. Avidano M, Singleton G. Adjuvant drug strategies in the treatment of recurrent respiratorypapillomatosis. Otolaryngol Hea d Neck Surg. 1995; 12:197-202

\section{CONFLICTOS DE INTERES}

Los autores declaran que no existe ningún conflicto de interés. 